\title{
Shoulder and Elbow Tendinitis as Initial Manifestation of Autoimmune Hepatitis
}

\author{
Huang Wei Ling* \\ MD Infectious disease, General Practice, Nutrition, Acupuncture and Pain Management Specialist, Medical \\ Acupuncture and Pain Management Clinic, Franca, São Paulo, Brazil
}

*Corresponding Author: Huang Wei Ling, MD Infectious disease, General Practice, Nutrition, Acupuncture and Pain Management Specialist, Medical Acupuncture and Pain Management Clinic, Franca, São Paulo, Brazil, Email: weilingmg@gmail.com

\begin{abstract}
Introduction: Tendinitis is commonly defined as an inflammation of the tendon, which is what connects bones to muscle. On the major literature, tendinitis is usually caused by repetition of movements and lack of stretching. However, the cause was never before associated to a disease in the liver, such as Autoimmune Hepatitis. According to Traditional Chinese Medicine (TCM), tendons are governed by the energy of the Gallbladder meridian, which is attached to the Liver energy.
\end{abstract}

Purpose: The aim of this study is to demonstrate that tendinitis may be an early manifestation of Autoimmune Hepatitis.

Methods: Through a clinical case report of a female patient, M.C., 42 years old, with chronic pain in shoulder and elbow, resistant to conventional treatments with anti-inflammatories and physiotherapy. She sought acupuncture treatment, and even so, the improvement presented was scarce. In her physical examination, a bulging was detected in the right ear, on the area that corresponds to the Liver, according to Auricular Medicine. Laboratory tests were requested to see if there could be any undiagnosed liver disease until then. The only exam that was initially altered was the prothrombin time of 89\%. More tests were ordered to see whether anti-bodies were present. Anti-nuclear, anti-smooth muscle, anti-microsomal.

Results: To the physician's surprise, the patient had a 1:160 anti-nucleus reagent.

Conclusion: The conclusion of this study is that tendinitis may be the initial manifestation of an Autoimmune Hepatitis.

Keywords: Tendinitis, Autoimmune Hepatitis, Traditional Chinese Medicine, Auricular Acupuncture, Liver.

\section{INTRODUCTION}

Tendinitis is defined as a process of inflammation of a tendon. In Western Medicine, is usually associated with repetition of movements and excess of use of one part of the body. For example, the wrists for those who play musical instruments, the knee for those who run, the ankle for those who play sports with feet [1-2].

The disease is also associated to incorrect posture and poor conditioning before exercise. [3] Overuse of the tendons is also associated to the cause. The symptoms are pain in the site of the tendon and surrounding area. The pain usually starts weak, and become suddenly severe. Another common symptom is loss of motion in the shoulder [1-2].
The treatment is based on the use of pain relievers and corticosteroids in the majority of times. Topical creams and other antiinflammatory medications are also very common. There are also specific programs of exercising in order to fortify the weakened tendon, diminishing the risk of relapses [1-2]. Tendinitis is a chronic condition, and will accompany those who acquired it, all the times the tendons are overused or used wrong. Therefore, Western Medicine see the exercises as a secure option [1-2].

In severe cases, surgical intervention may be required. Three procedures are used: Dry needling, ultrasonic treatment and surgery [1-2].

The dry needling consists in opening small holes in the tendon with a needle, to stimulate the 
tendon to heal, and release the inflammatory condition [1-2].

The ultrasonic treatment is a minimally invasive procedure that uses a small incision to insert a device removing the tissue scar tissues with ultrasonic sound waves.

Depending on the severity of the case, an actual surgery may be required as well. Especially when the tendon has torn away from the bone [1-2].

For those who have difficulty in changing functions and exercises, the tendinitis may be an everyday problem. As the usual treatments only aim to cure the symptoms, patients often seek for other traditions and comprehensions of the pathology, and many times, they will encounter Traditional Chinese Medicine as an option [4].

According to Traditional Chinese Medicine (TCM), tendons are governed by the energy of the Gallbladder meridian, which is attached to the Liver energy. Therefore, problems with the tendons will often be associated with problems in the Liver energy and in the organic level of the Liver as well [4].

In the case report described, the tendinitis flags a symptom in the Liver, Autoimmune Hepatitis. The patient received treatment for all her energy imbalances, according to the previous articles of the author, based on the case report of one specific patient in 2007 [5-6].

\section{Purpose}

The aim of this study is to demonstrate that tendinitis may be an early manifestation of Autoimmune Hepatitis.

It is also a goal to demonstrate how, in many cases, the focus of Western Medicine is only the symptoms, and the study and analysis of these symptoms, if not considering the patient holistically, and not taking into consideration the many interconnections between different organs, the hepatopathology would never be discovered, until it the Liver itself present symptoms.

As Hepatitis may take years to demonstrate symptoms, the pathology would only be discovered when the disease was already advanced.

\section{MeTHOdS}

The method used was a clinical case report of a female patient, M.C., 42 years old, with chronic pain in shoulder and elbow, divorced and with three children, working for three years in a company that sells insurance by telephone. She was facing a lot of anger in her work, making phone calls to clients while trying to sell insurance. As she called during business hours, where people are normally working, she received many rude replies. This caused a lot of weakening for her, and she would keep reviving and discussing these situations with co-workers, despite not being productive in her work.

The patient started feeling a severe pain in the right wrist, right elbow, and the picture has been progressively aggravating also affecting the right shoulder.

She first sought an orthopedist who advised physical therapy sessions and a non-hormonal anti-inflammatory medication, but the pain did not improve at all. For this reason, she sought the acupuncture procedure, where she was instructed to do two sessions per week, and despite the treatment, she obtained little improvement in pain. The doctor who was treating her with acupuncture, observed by placing the ear points in her ear that the area of the ear that corresponds to the Liver was presenting a bulging (Figure1), which might suggest some liver alteration.

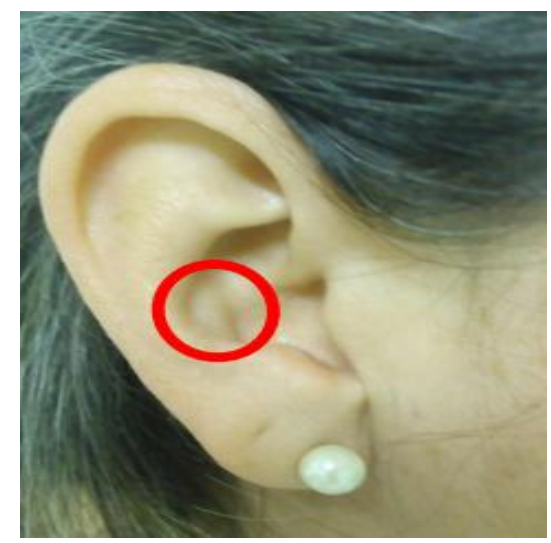

Figure1. Ear of the Patient

She suggested some initial laboratory tests to check for the existence of some inflammation or alteration in the liver function tests and/or the presence of virus of hepatitis B or C. No change was found other than a slightly extended prothrombin time of $89 \%$, showing a slight malfunction of the liver. Therefore, a few more tests were requested to see if there was any presence of antibodies or some autoimmune disease.

The patient started treatment with Chinese dietary counselling, auricular acupuncture associated to apex ear bloodletting and awareness to avoid the exposure to external pathogenic factors (Wind, Cold, Heat, Humidity 
and Dryness), in order to reduce the pain episodes.

Regarding the diet, the patient was advised to consume foods containing more liquids and avoid baked or grilled meat, in an attempt to get more Yin energy. She was also advised to avoid all foods that could be attacking the liver and gallbladder such as, fried foods, eggs, chocolate, honey, coconut, alcoholic beverage, and everything that had milk derivatives, especially when the cheese is melted. At last, she was also oriented to avoid soda, coffee and matte tea, who also weaken the Yin energy.

The patient was oriented regarding the influence of her negative emotions in her state of health, unbalancing the energy of the Liver, which would imbalance the Gall bladder (hollow organ of the Liver), the responsible organ for the tendons in Traditional Chinese Medicine. Understanding this process, the patient was able to control her emotions better.

\section{Results}

The bulging in the patient's ear, when analysed trough the perspective of auricular acupuncture denotated some alteration in the Liver meridian, when examined, the result of the test came positive for several anti-bodies (anti-positive nuclear body 1/180).

After discovering the negative influence of anger in her competitive work environment, the patient began to deal more peacefully with situations, being able to learn how to work in a more productive way, without complaining to her work colleagues the various negative situations that happened to each call. This behavior improved her performance. As she gains over what she sells, this had a positive effect in her personal life. With less anger episodes, the patient was able to diminish the episodes of pain as well.

\section{DISCUSSION}

\subsection{Autoimmune Hepatitis and Western Medicine}

The autoimmune hepatitis is understood as a situation where the immune systems rises against the liver cells. The reason behind this behavior of the immune system is still unclear and genetic and environmental factors are associated with the causes.

When not treated the disease my lead to cirrhosis and liver failure, when early diagnosed, the disease can be controlled. As the disease is asymptomatic, it is common for the acute phase to pass undiagnosed. The symptoms, when they appear, are fatigue, abdominal discomfort, yellowing of the skin and eyes, and enlarged liver, abnormal blood vessels on the skin, skin rashes, joint pain, and loss of menstrual periods. [7-10]

There are some risk factors to have the disease and they are: Being female, a history of certain infections, hereditary problems with the disease, having one or more autoimmune diseases before. [7-10]

There are two types of autoimmune hepatitis, according to Western Medicine, the type 1, is the most common type, it can occur in any age, and the majority of the patients are facing other autoimmune diseases in conjunction to hepatitis. [7-10]

Type 2 is more common in children and young people, mainly girls. Although it may also be accompanied by other autoimmune disorders, it appears alone in the majority of the cases. [7-10]

The autoimmune hepatitis nowadays, can be treated with the daily use of medications, in a different amount time regarding the seriousness of the disease, the treatment might have weeks or months. [7-10]

If not treated in time, the autoimmune hepatitis can develop many other issues, such as enlarged veins in your esophagus, fluid in the abdomens, liver failure and liver cancer [7-10].

\subsection{Traditional Chinese Medicine Main Principles}

There are substantial differences between Traditional Chinese Medicine and Western Medicine perspectives, and is very important to understand these two ways of seeing and treating patients are not opposite, but complementary. [11]

With these two perspectives working together, many of the TCM tools can be used to diagnosed pathologies well know in Western Medicine, and in the case of autoimmune hepatitis, asymptomatic in the majority of the time when the disease is developing and growing inside the body. Being able to identify pathology, still in the energy level can be extremely useful for all the medicine nowadays.

The author published one article regarding autoimmune hepatitis, entitled Can Autoimmune Hepatitis Be Treated Without the Use of Corticosteroids and Immunosuppressive 
Drugs?. In the case report of the patient of this study, the same ideas were used, treating the energy imbalances of the patient and avoiding the use of high-concentrated drugs, that could maintain an unhealthy cycle, worsening the energy imbalances inducing the autoimmune disease. [12]

In order to understand Traditional Chinese Medicine and see how the diagnosis of that patient was done, it is important to comprehend the main principles of Traditional Chinese Medicine. [11]

\subsubsection{Yin and Yang}

It is impossible to understand Traditional Chinese Medicine (TCM) without understanding the main concepts of Yin and Yang. This concepts base everything in which Traditional Chinese Medicine believes in terms of treatment and cure of pathologies. [11]
Yin and Yang are energy forces that rule the world. Everything in the universe is composed by Yin and Yang, including our body (Figure 2). [11]

The Chinese wise men represented this complement of opposites by the archetypal pair, Yin and Yang, considering their dynamic interaction as the essence of all-natural phenomena and all human situations. Opposites are complementary. To be healthy, we aim for a state of equilibrium between Yin and Yang energies. If there are imbalances between these two energies, symptoms occur, and if not properly treated, consolidates the disease in the future. [11]

Autoimmune diseases are associated with Yin deficiency, according to Traditional Chinese Medicine [13]

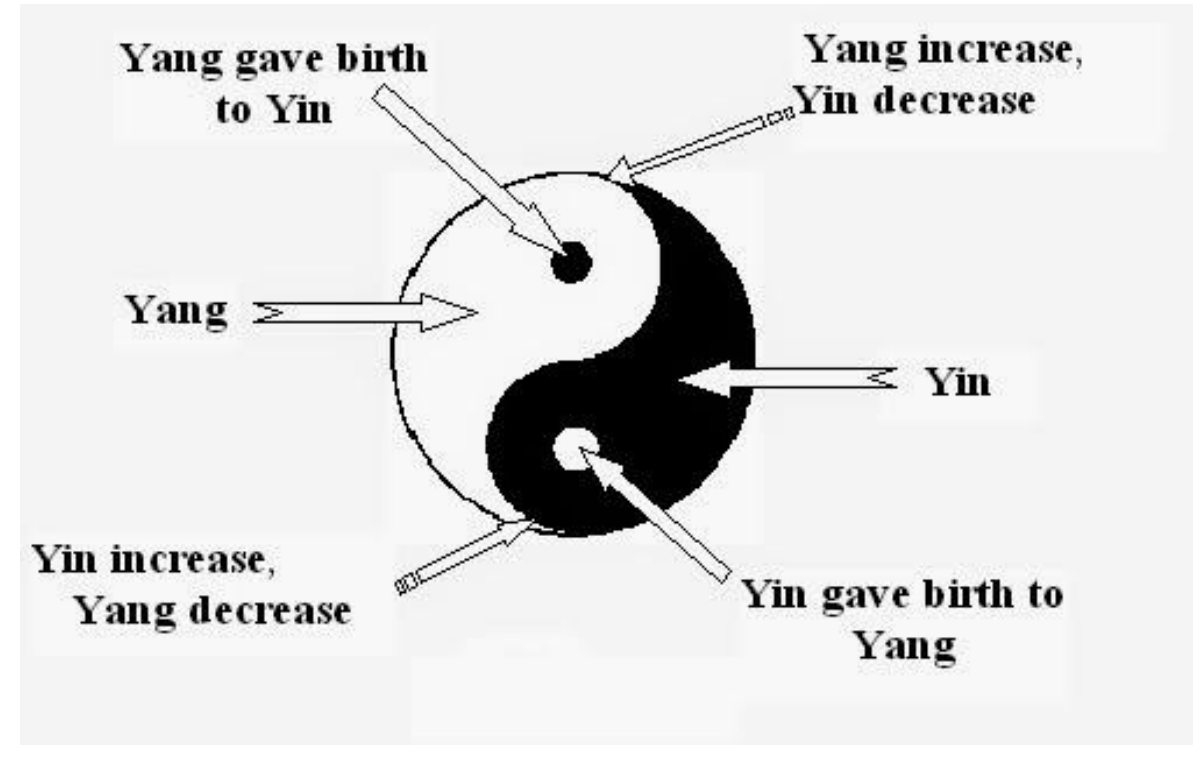

Figure2. Yin and Yang scheme

In the case of the patient described, the tendonitis demonstrated a relationship with the Liver meridian that has the Gall bladder as hollow organ, responsible for the tendons in TCM.

\subsubsection{Five Elements Theory}

The second main theory basing Traditional Chinese Medicine is the Five Elements theory. Regarding the body, this is the most important theory presented by the Oriental traditions, primordial to understand the diagnosis of the patient in the case report. [11]

Five Elements theory states that there are five elements present in everything in the world, including our bodies. These elements are Fire, Earth, Metal, Water and Wood [11].
Inside our bodies, these elements will be represented by specific organs of our body. These organs are considered to be the main organs, and they have extremely important functions in the well-functioning of the body.

They are completely interconnect, as the main principle of the Five Elements theory is to understand the idea of cycle, previously presented in the Yin and Yang section. [11]

The Wood element corresponds to the Liver, The Fire element corresponds to the Heart. The Earth element corresponds to the Spleen, The Metal element corresponds to the Lungs, and the Water element corresponds to the Kidney, as represented in Figure3 [11]. 


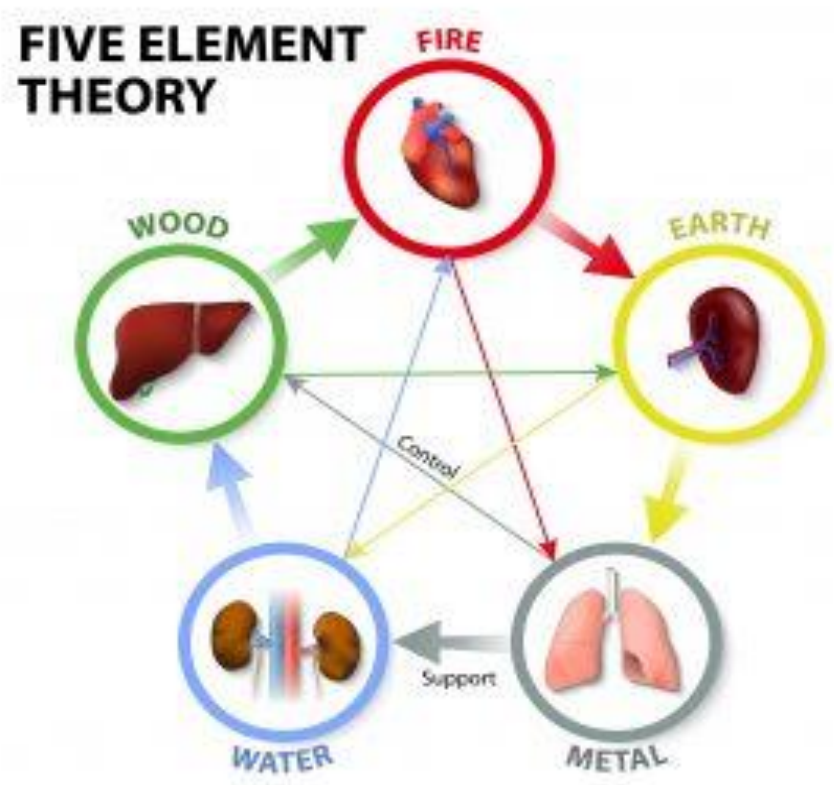

Figure3. Five Elements Theory

These main organs will support and control each other in the same cycle presented by the Five
Elements. Each massive organ has a hollow organ, as described in Table 1.

Table1. Five Elements Theory and Correspondences

\begin{tabular}{||c|c|c|c|c|c||}
\hline \multicolumn{9}{||c|}{} & \multicolumn{2}{l||}{} \\
\hline \hline & WATER & WOOD & FIRE & EARTH & METAL \\
\hline \hline Season & Winter & Spring & Summer & Late Summer & Autumn \\
\hline \hline Climatic Qi & Cold & Wind & Heat & Damp & Dryness \\
\hline \hline Yang Organ & Bladder & Gallbladder & Sm. Intestine & Stomach & Lg. Intestine \\
\hline \hline Yin Organ & Kidney & Liver & Heart & Spleen & Lung \\
\hline Sense Organ & Ears & Eyes & Tongue & Mouth & Nose \\
\hline \hline Body Tissue & Bone & Sinews & Blood Vessel & Muscles & Skin \\
\hline \hline Emotion & Fear & Anger & Joy/Shock & Worry & Sadness \\
\hline \hline Color & Black & Green & Red & Yellow & White \\
\hline \hline Taste & Salty & Sour & Bitter & Sweet & Spicy \\
\hline \hline
\end{tabular}

Liver has the eye as external sensory organ, is responsible for vision, and has the Gall bladder as its hollow organ. Heart is responsible for the tongue, and subsequently language and has the Small Intestine as its hollow organ. Spleen represents the mouth, is responsible for taste, and has the Stomach as its hollow organ. Lungs have the nose as external sensory organ, are responsible for the sense of smell, and have the Large Intestine as its hollow organ. Kidney represents the ear, is responsible for the sense of hearing, and has the bladder as its hollow organ [11].

This information is important for the physician, because the visible symptoms of external sensorial organs are just the tip of the iceberg. The cause, which is in the root, may be found through this relation. In the case of the patient described in the case report, she had a symptom in the tendons, associated with the Gall Bladder and therefore with the Liver, together with the bulging found in her ear. It is very important to search for the real root of the problem instead of only treating the symptoms.

\subsection{Auricular Acupuncture: Reflexology and the Liver Point}

The Auricular Acupuncture is technique developed on the West Europe, although based on the Acupuncture techniques, developed on the Asian countries, 2000 years ago. It was 
better developed by the German physicians, with associated the points on the ear as a microsystem of the whole body, being able to diagnose and treat using it. [14]

The major idea of the auricular acupuncture is that the ear has a representation of an inverted fetus, and on this fetus, we have all the anatomical properties of the body. As the ear is formed first, on the 18th week of pregnancy, it is believed that it developed a microsystem, representing the body. [14]

This theory coincides with the theory presented by the Canadian neurologist, Paul Nogier, which defended the idea of a map of the body existing on the surface of the brain. This map would spread to different areas of the body, developing microsystems. The ear and the feet are two good examples. This idea was able to explain on other words, the exactly treatment done by TCM. [15]

Anatomically, the ear has three parts: the external ear, the middle ear and the internal ear. The external ear has the external auditory canal and the auricle. On this area, there are four prominences, three depressions and four notches. Besides the ear lobe on the anterior surface of the auricle [14]. On Traditional Chinese Medicine, all this points and divisions of the parts of the ear were associated with specific parts of the body, containing all the anatomical parts of the total body. [14]

Deepen the knowledge on the Auricular Acupuncture studies, it is possible to better understand and diagnosis a range of diseases and other points which may be helpful on different situations and disorders. [14]

On the specific case of the patient on this case report, she had two symptoms of a liver disorder in Traditional Chinese Medicine: a bulging on the area corresponding to the Liver as well as pain in the tendons, diagnosed as tendonitis by orthopedists, which leaded for the test and diagnosis of autoimmune hepatitis, as the root of the problem.

Acupuncture is specialty recognized by the Federal Medical Council in Brazil since 1995. $[5-6,12]$

\subsection{Arndt-Schultz's Law}

The author, in her daily practice, noticed that acupuncture was a very useful tool, but had limits. When the patient, as in the case report described, had deep energy deficiencies, the use of acupuncture had to be complemented with medication to restore these energies. [5-6]

According to the Arndt-Schultz law (Figure 4), high-concentrated medication can be harmful to health, reducing vital energy, and therefore, creating Internal Heat retention, according to TCM reasoning. Based on this idea, during the patient's treatment, the author tried to avoid the use of highly concentrated drugs, even in the treatment of acute pathologies, such as sore throat and respiratory tract infections, common in childhood. [5-6]

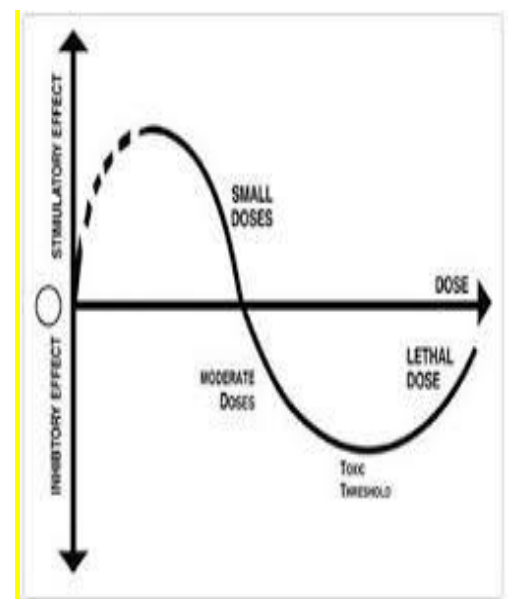

Figure4. Arndt-Schultz's Law

The main purpose of avoiding these drugs was to avoid worsening the patient's energy level and not create more Heat retention, responsible for his emotional distress.

A second explanation is that the medicine itself can directly attack the energy of the liver leading to the disharmony of the energy of the Gallbladder and consequently, worsening inflammation of the tendons. [4]

The patients in the case report were taking medication for the tendonitis symptoms when the treatment started. According to the ArndtSchultz law, the intake of those medications was harming the vital energy of the patient, creating more formation of internal Heat, leading to more Yin deficiency, creating an unhealthy cycle that would maintain or worse the autoimmune disease the patient already had.

\subsection{Yin and Yang Metaphor of Western and Traditional Chinese Medicine}

The treatment of the patient described in the case report is one example of the possibility of an integral perspective between Western and Traditional Chinese Medicine. The body, and therefore, the possible treatments, is not only 
physical and constituted by organs and tissues. It is formed by energy. In Figure 5, a metaphor of Yin and Yang as the different kinds of medicine was created by the author. Nowadays, these two perspectives are separated, but they are different but can be complementary. One cannot exist without the other. [5-6]

Traditional Chinese medicine can be considered as Yang energy, and Western medicine is considered Yin energy, already materialized. In the same way of the relationship of the Yin and Yang, Traditional Chinese Medicine starts where Western Medicine ends, and the opposite is also true. The relationship is not static, but dynamic. [5-6]

In the treatment of tendonitis, in this specific case, it was important to have an integral view, using both medical perspectives, for a deeper and integral problem of the real cause of the problem in the energy level.

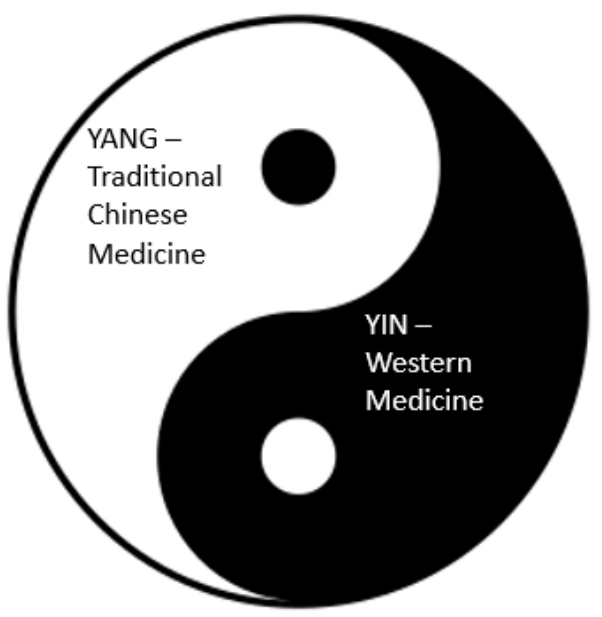

Figure5. Traditional Chinese Medicine and Western Medicine - Yin and Yang Metaphor

\section{CONCLuSion}

The conclusion of this study is that autoimmune hepatitis can manifest itself in several ways, and in this study, we demonstrate that it can be started with the case of tendinitis. According to traditional Chinese medicine, the internal organ that is responsible for the tendons is the energy of the Gallbladder, which is an organ attached to the Liver. With the Liver unbalanced, the Gallbladder will also be affected, inflaming the tendons.

Therefore, patients presenting tendinitis should generally be investigated if they have any Liver or Gallbladder disease, either from a laboratorial point of view through examinations or from an energetic point of view, according to Traditional Chinese Medicine.

\section{REFERENCES}

[1] Almekinders LC, Temple JD. Etiology, diagnosis, and treatment of tendonitis: an analysis of the literature. Med Sci Sports Exerc. 1998 Aug; 30(8):1183-90.

[2] Federer AE, Steele JR, Dekker TJ, Liles JL, Adams SB. Tendonitis and Tendinopathy: What Are They and How Do They Evolve? Foot Ankle Clin. 2017 Dec;22(4):665-676. doi: 10.1016/j.fcl.2017.07.002. Epub 2017 Sep 27.

[3] DeokJu Kim, MiLim Cho, YunHee Park, and YeongAe Yang. Effect of an exercise program for posture correction on musculoskeletal pain. J Phys Ther Sci. 2015 Jun; 27(6): 1791-1794. Published online 2015 Jun 30. doi: 10.1589/ jpts.27.1791

[4] Chia-Man Ma, Lih-Hwa Lin, Yung-Hsiang Chen, Huey-Yi Chen, Jen-Huai Chiang, and Wen-Chi Chen. Liver Governs Tendon: A Theory from Traditional Chinese MedicineEvidence from a Population-Based Matched Cohort Study in Taiwan for the Association of Chronic Liver Disease and Common Diseases in the Chiropractic Office. Evidence-Based Complementary and Alternative Medicine Volume 2016, Article ID 7210705, 8 pages. http://dx.doi.org/10.1155/2016/7210705

[5] Ling HW (2019) Is it Possible to Treat Community-Acquired and Nosocomial Infections with the Same Method, Without the Use of Antibiotics? J Appl Microb Res. Vol: 2 Issu: 2 (01-13).

[6] Huang WL (2019) Is It Possible to Treat Giant Congenital Hairy Melanocytic Nevus Clinically? J Clin Case Rep Trials. Vol: 2, Issu: 2 (06-13)

[7] Albert J. Czaja. Diagnosis and Management of Autoimmune Hepatitis: Current Status and Future Directions. Gut Liver. 2016 Mar; 10(2): 177-203. Published online 2016 Mar 15. doi: $10.5009 /$ gnl15352

[8] Dhruv Lowe and Savio John. Autoimmune hepatitis: Appraisal of current treatment guidelines. World J Hepatol. 2018 Dec 27; 10(12): 911-923. Published online 2018 Dec 27. doi: $10.4254 /$ wjh.v10.i12.911

[9] Sebode M, Hartl J, Vergani D, Lohse AW. Autoimmune hepatitis: From current knowledge and clinical practice to future research agenda. Liver Int. 2018 Jan;38(1):1522. doi: 10.1111/liv.13458. Epub 2017 May 18.

[10] Liberal R, Grant CR, Mieli-Vergani G, Vergani D. Autoimmune hepatitis: a comprehensive review. J Autoimmun. 2013 Mar; 41:126-39. 
doi: 10.1016/j.jaut.2012.11.002. Epub 2012 Dec 4.

[11] OuYang B, Gu Z (1996) Essentials of Traditional Chinese Medicine. Shandong, Science and Technology Press

[13] Wanshu Hou, Guangpi Xu and Hanjie Wang. Treating Autoimmune Disease with Chinese Medicine. Churchill Livingstone Elsevier Publisher.

[14] Li-Chun Huang, Auricular Medicine: The New Era of Medicine and Healing.
[12] Huang WL. "Can Autoimmune Hepatitis Be Treated Without the Use of Corticosteroids and Immunosuppressive Drugs?" Acta Scientific Medical Sciences 3.7 (2019): 178-186.

[15] Gerhard Litscher,Theodoros Yannacopoulos, and Peter Kreisl. Nogier Reflex: Physiological and Experimental Results in Auricular Medicine-A New Hypothesis. Medicines (Basel). 2018 Dec; 5(4): 132. Published online 2018 Dec 12. Doi: 10.3390/medicines5040132

Citation: Huang Wei Ling, Shoulder and Elbow Tendinitis as Initial Manifestation of Autoimmune Hepatitis. ARC Journal of Orthopedics. 2019; 4(2):19-26. doi:dx.doi.org/10.20431/2456-0588.0402004.

Copyright: (C) 2019 Authors. This is an open-access article distributed under the terms of the Creative Commons Attribution License, which permits unrestricted use, distribution, and reproduction in any medium, provided the original author and source are credited. 